\section{Is ATP Elevated in Patients With GAMT Deficiency?}

We read with interest the paper by Bianchi et al, ${ }^{1}$ in which the authors report on the treatment monitoring of 2 patients with guanidinoacetate methyltransferase (GAMT) deficiency and 3 patients with an arginine:glycine amidinotransferase defect (AGAT-d). Repetitive MR measurements of these patients are not trivial, and some interesting observations are presented in this paper that may be very useful for their treatment.

Bianchi et $\mathrm{al}^{1}$ studied the levels of brain creatine (Cr) and phosphocreatine in 1 patient with GAMT deficiency using ${ }^{31} \mathrm{P}$ - and ${ }^{1} \mathrm{H}-\mathrm{MR}$ spectroscopy (phosphorous and proton MR spectroscopy) before and after therapy. The authors conclude from the ${ }^{31} \mathrm{P}-\mathrm{MR}$ spectra that levels of adenosine triphosphate (ATP) are elevated in the brain of this patient before therapy, and that these levels normalize with treatment. Furthermore, they speculate that this may be the result of redistribution in the creatine kinase $(\mathrm{CK})$ reaction $(\mathrm{ATP}+\mathrm{Cr}$ - ADP + PCr) from the reduced levels of $\mathrm{Cr}$ in the brains of other patients. ${ }^{1}$ In our opinion, this conclusion on increased ATP and the speculation on its origin are debatable.

Tissue levels of ATP are tightly balanced by consumption and production of ATP. Because the brain is a highly oxidative organ, most production of ATP occurs by mitochondrial oxidative phosphorylation, though other processes such as glycolysis also contribute. The primary function of the $\mathrm{CK}$ reaction is more to dampen large fluctuations in the ratios of ATP and adenosine diphosphate (ADP) during periods of high energy demand, ${ }^{2}$ all in close relationship to the energy-producing and energy-consuming sites. Therefore, it is unlikely that a mere shift in the concentration of $\mathrm{Cr}$ will result in the drastic, global elevation of ATP as reported.

According to Bianchi et al, ${ }^{1}$ the supposed elevation of ATP is based on the ${ }^{31} \mathrm{P}-\mathrm{MR}$ spectrum of GAMT; in patient 1 , in particular, the signal intensity for $\beta$-ATP (at $16.5 \mathrm{ppm}$ ) is increased. However, ATP has 3 phosphate groups, all giving a distinct signal intensity at different positions on the ${ }^{31} \mathrm{P}-\mathrm{MR}$ spectrum. If ATP were elevated, why are not all 3 signals equally elevated, since they belong to the same molecule? Although the other ATP signals ( $\alpha$-ATP and $\gamma$-ATP) also represent other phosphorus compounds such as ADP, these are present at much lower concentrations $(\sim \mu \mathrm{M}$ range) than ATP $(\sim \mathrm{mM})$. Therefore, changes in the levels of these compounds cannot account for a selective elevation in the signal intensity of $\beta$-ATP. We believe that the increase in the signal intensity of $\beta$-ATP may be because of experimental issues in the ${ }^{31} \mathrm{P}-\mathrm{MR}$ spectroscopy method. Factors such as incorrect phasing of the MR spectrum, the absence of a firstorder phase correction, and insufficient bandwidth of the excitation pulse in combination with a misplaced carrier resonance could all play a role here. Unfortunately, Bianchi et $\mathrm{al}^{1}$ did not provide sufficient information on data acquisition and quantification of the ${ }^{31} \mathrm{P}$-MR signals to resolve this issue.

Finally, the authors state that my colleagues and $\mathrm{I},{ }^{3}$ in GAMTdeficient mice, and Schulze et $\mathrm{al}^{4}{ }^{4}$ in a patient, display the same abnormality in the signal intensity of ATP and that both my colleagues and I and Schulze et al failed to provide a comment on this. However, in our opinion, our ${ }^{31} \mathrm{P}$-MR spectra of the GAMT-deficient mouse brain did not reveal a similar abnormality in the signal intensity of $\beta$-ATP, ${ }^{3}$ as Bianchi et $\mathrm{al}^{1}$ observed. As indicated in the legend to Fig 6 of our paper, the ${ }^{31} \mathrm{P}-\mathrm{MR}$ spectra are scaled arbitrarily. Also, in the paper of Schulze et al, ${ }^{4}$ it seems that signal intensity is scaled arbitrarily. At least when the reference signal intensity (ie, phosphodiester signal intensity) applied by Bianchi et $\mathrm{al}^{1}$ is used, no significant change in the signal intensity of ATP is observed. Therefore, in our opinion, the ATP increase in GAMT deficiency before treatment with Cr is not supported by other studies.

\section{References}

1. Bianchi MC, Tosetti M, Battini R, et al. Treatment monitoring of brain creatine deficiency syndromes: a $1 \mathrm{H}$ - and ${ }^{31} \mathrm{P}-\mathrm{MR}$ spectroscopy study. AJNR Am J Neuroradiol 2007;28:548-54

2. Wyss M, Smeitink J, Wevers RA, et al. Mitochondrial creatine kinase: a key enzyme of aerobic energy metabolism. Biochim Biophys Acta 1992;1102: 119-66

3. Renema WK, Schmidt A, van Asten JJ, et al. MR spectroscopy of muscle and brain in guanidinoacetate methyltransferase (GAMT)-deficient mice: validation of an animal model to study creatine deficiency. Magn Reson Med 2003;50:936-43

4. Schulze A, Hess T, Wevers R, et al. Creatine deficiency syndrome caused by guanidinoacetate methyltransferase deficiency: diagnostic tools for a new inborn error of metabolism. J Pediatr 1997;131:626-31

W.K.J. Renema

H.E. Kan

A. Heerschap

Department of Radiology

UMC St Radboud

Nijmegen, the Netherlands

DOl 10.3174/ajnr.A0803

\section{Reply:}

On behalf of all of the authors of our study, ${ }^{1}$ we thank Dr Renema for his interest in our work and we appreciate his comments. We are fully aware that other studies demonstrate that the cellular pools of adenosine triphosphate (ATP) are very stable during tissue activation and that they are regulated not only by the creatine kinase (CK) system but also by mitochondrial oxidative phosphorylation, glycolysis, and the adenosine monophosphate-cyclase system. Most of these studies have been performed on muscles, and only few data are available for the brain in which the control, coupling, and kinetics of the phosphocreatine/CK/ATP system are much more complex. ${ }^{2,3}$ Acquisition and postprocessing of our ${ }^{31} \mathrm{P}-\mathrm{MR}$ spectroscopy studies were carried out according to standard procedures for human brain studies. The increased signal intensity of the ATP peaks in the brain of the child with the guanidinoacetate methyltransferase defect (GAMT-d; Fig 2 of our original article) was unique. Due to the different arbitrary units used by Renema et $\mathrm{al}^{2}$ and Schulze et $\mathrm{al},{ }^{4}$ we have probably erroneously referenced these articles as supporting our results. Therefore, we believe that the question raised by Renema is still waiting to be confirmed by further ${ }^{31} \mathrm{P}-\mathrm{MR}$ spectroscopy studies of patients with GAMT-d aimed at measuring absolute concentration of brain ATP.

\section{References}

1. Bianchi MC, Tosetti M, Battini R, et al. Treatment monitoring of brain creatine deficiency syndromes: a $1 \mathrm{H}$ and ${ }^{31} \mathrm{P}-\mathrm{MR}$ spectroscopy study. AJNR Am J Neuroradiol 2007;28:548-54

2. Renema WK, Schmidt A, van Asten JJ, et al. MR spectroscopy of muscle and brain in guanidinoacetate methyltransferase (GAMT)-deficient mice: validation of an animal model to study creatine deficiency. Magn Reson Med 2003;50:936-43

3. Schmidt A, Marescau B, Boehm EA, et al. Severely altered guanidino compound levels, disturbed body weight homeostasis and impaired fertility in a mouse model of guanidinoacetate N-methyltransferase (GAMT) deficiency. Hum Mol Genet 2004;13:905-21

4. Schulze A, Bachert P, Schlemmer $\mathrm{H}$, et al. Lack of creatine in muscle and brain in an adult with GAMT deficiency. Ann Neurol 2003;53:248-51

M.C. Bianchi

DOI 10.3174/ajnr.A0805 